\title{
Early stage of integrable turbulence in the one-dimensional nonlinear Schrödinger equation: A semiclassical approach to statistics
}

\author{
Giacomo Roberti, ${ }^{1}$ Gennady El, ${ }^{1}$ Stéphane Randoux, ${ }^{2,3}$ and Pierre Suret $\odot^{2,3, *}$ \\ ${ }^{1}$ Department of Mathematics, Physics and Electrical Engineering, Northumbria University, Newcastle upon Tyne, NE1 8ST, United Kingdom \\ ${ }^{2}$ University of Lille, CNRS, UMR 8523 - Physique des Lasers Atomes et Molécules (PHLAM), F-59000 Lille, France \\ ${ }^{3}$ Centre d'Etudes et de Recherches Lasers et Applications (CERLA), Cité scientifique, 59655 Villeneuve d'ascq Cedex, France
}

(Received 8 January 2019; published 19 September 2019)

\begin{abstract}
We examine statistical properties of integrable turbulence in the defocusing and focusing regimes of onedimensional small-dispersion nonlinear Schrödinger equation (1D-NLSE). Specifically, we study the 1D-NLSE evolution of partially coherent waves having Gaussian statistics at time $t=0$. Using short time asymptotic expansions and taking advantage of the scale separation in the semiclassical regime we obtain a simple explicit formula describing an early stage of the evolution of the fourth moment of the random wave field amplitude, a quantitative measure of the "tailedness" of the probability density function. Our results show excellent agreement with numerical simulations of the full 1D-NLSE random field dynamics and provide insight into the emergence of the well-known phenomenon of heavy (respectively, low) tails of the statistical distribution occurring in the focusing (respectively, defocusing) regime of 1D-NLSE.
\end{abstract}

DOI: 10.1103/PhysRevE.100.032212

\section{INTRODUCTION}

Turbulence is one of the most recognizable forms of nonlinear motion that has been, and continues to be, the subject of very active research in classical (viscous) fluid dynamics [1]. This fundamental phenomenon occurs also in dispersive media where turbulence is associated with the generation of complex, spatiotemporal statistical ensembles of interacting nonlinear waves. The theory of weak wave turbulence in dispersive systems was developed by V. E. Zakharov in the 1960s [2]. The wave turbulence (WT) theory provides a framework for the statistical description of weak turbulence in nonintegrable wave systems dominated by resonant interactions. One of the most important results in wave turbulence theory is the discovery by V. E. Zakharov in 1965 of a new type of solutions to kinetic equations corresponding to a constant energy flux through scales. These solutions are called Kolmogorov-Zakharov spectra, and they have been observed in a variety of experiments performed in turbulent wave systems [3].

The notion of turbulence can be extended to the realm of integrable systems where it is understood as complex spatiotemporal dynamics of nonlinear random waves in physical systems whose behavior is well modeled by integrable nonlinear partial differential equations such as the Kortewegde Vries (KdV) equation or the one-dimensional nonlinear Schrödinger equation (1D-NLSE). Since many nonlinear

\footnotetext{
*Corresponding author: Pierre.Suret@univ-lille.fr
}

Published by the American Physical Society under the terms of the Creative Commons Attribution 4.0 International license. Further distribution of this work must maintain attribution to the author(s) and the published article's title, journal citation, and DOI. wave systems can be described by partial differential equations having an integrable core part the emerging theory of integrable turbulence, also initiated by Zakharov [4], has become an active field of research with the theoretical (mostly numerical) developments supported by a number of experimental observations [4-14]. Given the absence of resonances in integrable systems, the mechanisms underlying integrable turbulence are of profoundly different nature from those found in the standard WT $[2,3,15]$ and thus require very different theoretical approaches to their study.

General properties of integrable wave systems can be in principle analyzed using the inverse scattering transform (IST) method [16], which thus provides a powerful analytical framework for the description of integrable turbulence. However, only very few analytical results are available in this direction due to the high complexity of the IST with random potentials, so alternative approaches based on, e.g., asymptotic expansions could prove very valuable. In this paper, such an asymptotic approach is developed in application to integrable turbulence in the framework of 1D-NLSE which plays a fundamental role in nonlinear physics due to the unique combination of complete integrability and the ubiquity in a broad range of applications.

Depending on the relative signs of dispersion and nonlinearity the 1D-NLSE can exhibit focusing or defocusing properties which have strong effect on the evolution of the statistics of random solutions. Generally speaking, nonlinear wave propagation in media with self-focusing nonlinearity tends to produce heavy-tailed deviations from the initial Gaussian statistics, observed in the probability density function (PDF) of the random wave field amplitude. In recent years, the question of the emergence of heavy-tailed statistical distributions has been extensively studied in relation to the occurrence of extreme events such as rogue waves, mainly 
in the physical contexts of fluid dynamics [17-20] and optics [7,8,14,21-25]. Even though statistical properties of nonlinear defocusing media have been less extensively examined, several experiments have shown that defocusing nonlinearities tend to produce low-tailed deviations from the initial Gaussian statistical distribution [26,27].

The "heaviness" of the tail in the PDF of the field amplitude distribution is characterized by the kurtosis (the fourth standardized moment of the PDF). In the WT setting, the theory based on the derivation of quasikinetic equations for the lowest-order moments of the wave field has been developed in Refs. [15,28-30]. This theory has confirmed that the kurtosis increases in the focusing case and decreases in the defocusing case while the initial field is characterized by Gaussian statistics. However, this kind of analytical treatment is inherently limited to the weakly nonlinear propagation regime.

To evaluate kurtosis for certain regimes of the fully nonlinear integrable turbulence in the 1D-NLSE one can take advantage of the mathematical framework of dispersive hydrodynamics - the semiclassical theory of nonlinear dispersive waves [31]. The definitive feature of dispersive hydrodynamics is the presence of two distinct spatiotemporal scales: the long scale specified by initial conditions and the short scale by the internal coherence length (i.e., the typical size of the coherent soliton-like structures). This scale separation enables one to analyze the wave evolution asymptotically.

The semiclassical, dispersive hydrodynamic approach describes the propagation regimes of a completely opposite nature compared to the regimes considered in the framework of wave turbulence theory. This approach can be applied to the 1D-NLSE propagation if the initial scale of the fluctuations of the power of the complex field $|\psi|^{2}$ are much larger than the one corresponding to the balance between nonlinearity and dispersion. In most of the standard cases, this separation of scales corresponds to situations where the nonlinear part of the energy is much greater that the linear (kinetic) part of the energy at the initial time. As shown in Ref. [32], this scale separation permits one to split the development of integrable turbulence into two distinct stages characterized by qualitatively different dynamical and statistical features. At the initial (we shall call it "prebreaking") stage of the evolution nonlinear effects dominate linear dispersion and the wave fronts of the random initial field experience gradual steepening leading to the formation of gradient catastrophes that are subsequently regularized through the generation of dispersive shock waves in the defocusing regime [33] and of Peregrine-like breather sequences in the focusing regime [34]. As shown in Ref. [32], the dynamical and statistical features that occur at the prebreaking stage of the defocusing 1D-NLSE can be interpreted in terms of the evolution of random Riemann waves.

In this paper, we extend the analysis of the previous works based on the semiclassical approach to the 1D-NLSE with random initial data by calculating the short-time evolution of the the normalized fourth moment $\kappa_{4}$ of the amplitude of the field. Similarly to the standard kurtosis, the quantity $\kappa_{4}$ describes the degree of the deviation from the initial statistical distribution which is often assumed to be Gaussian [35]. Using the semiclassical Madelung transform and performing the zero dispersion limit, we derive a general analytical expression for the short-time evolution of the fourth moment of the random 1D-NLSE wave field in terms of hydrodynamic variables, and show that this expression can be further simplified for the wave field having Gaussian statistics at initial time. Our analytical asymptotic results are shown to be in excellent agreement with numerical simulations of the evolution of partially coherent initial data in 1D-NLSE.

The paper is organized as follows: In Sec. II, using the semiclassical approximation, we identify the initial stage of the 1D-NLSE development of partially coherent waves with the nonlinearity dominated, dispersionless regime and derive the general expression for the short-time evolution of the fourth-order moment $\kappa_{4}$ as a power-series expansion in time $t$.

In Sec. III, we apply the derived formula for $\kappa_{4}$ to the fundamental case of random waves characterized by Gaussian statistics at time $t=0$. In Sec. IV we provide a comparison between our semiclassical analytical results and numerical simulations of 1D-NLSE.

\section{THE DISPERSIONLESS LIMIT OF THE 1D-NLSE AND THE TIME EVOLUTION OF THE FOURTH-ORDER MOMENT OF A RANDOM WAVE FIELD}

We consider the 1D-NLSE in the normalized form

$$
i \varepsilon \frac{\partial \psi}{\partial t}+\frac{\varepsilon^{2}}{2} \frac{\partial^{2} \psi}{\partial x^{2}}+\sigma|\psi|^{2} \psi=0,
$$

where $\psi$ is a complex field, $\varepsilon$ is the dispersion parameter, $\sigma=-1$ in the defocusing regime, and $\sigma=+1$ in the focusing regime.

The 1D-NLSE (1) is considered in a periodic box of size $L$, $\psi(x+L, t)=\psi(x, t) \forall t$. The field $\psi$ then can be represented as a Fourier series:

$$
\psi(x, t)=\sum_{k} \psi_{k}(t) e^{\frac{2 i \pi}{L} k x} \quad \text { with } \quad k \in \mathbb{Z},
$$

where the Fourier coefficients are given by

$$
\psi_{k}(t)=\frac{1}{L} \int_{0}^{L} \psi(x, t) e^{-2 i \pi k x} d x .
$$

The "density of particles" $N$ and the momentum $P$ represent integrals of motion and are expressed in terms of Fourier coefficients:

$$
\begin{gathered}
N=\frac{1}{L} \int_{0}^{L}|\psi|^{2} d x=\sum_{k}\left|\psi_{k}\right|^{2}, \\
P=\frac{1}{L} \int_{0}^{L} \psi_{x} \psi^{*} d x=\sum_{k}\left(\frac{2 \pi i k}{L}\right)\left|\psi_{k}\right|^{2} .
\end{gathered}
$$

The Hamiltonian, that we represent in the form

$$
H=\varepsilon^{2} H_{L}+H_{\mathrm{NL}}
$$

is also integral of motion, which is naturally split into two parts: the linear (kinetic energy) part,

$$
\varepsilon^{2} H_{L}(t)=\frac{\varepsilon^{2}}{2 L} \int_{0}^{L}\left|\psi_{x}\right|^{2} d x=\frac{\varepsilon^{2}}{2} \sum_{k}\left(\frac{2 \pi k}{L}\right)^{2}\left|\psi_{k}\right|^{2},
$$


and the nonlinear part,

$$
H_{\mathrm{NL}}(t)=\frac{\sigma}{2 L} \int_{0}^{L}|\psi|^{4} d x
$$

We now assume that the Fourier modes at initial time $\psi_{0 k}=\psi_{k}(t=0)=\left|\psi_{0 k}\right| e^{i \phi_{0 k}}$ are complex random variables. The complex field (2) is then a random periodic solution of the 1D-NLSE. No particular hypothesis about statistical properties of $\psi_{0 k}$ needs to be introduced at this step but we will show in Sec. III that the main result of our analysis can be simplified if the initial statistics of the random wave field is assumed to be Gaussian. We consider random initial conditions for which $N, H_{L}(t=0)$ and $H_{\mathrm{NL}}(t=0)$ are all $\mathcal{O}(1)$. This is typically achieved by taking the initial power spectrum $n_{0 k}=\left|\psi_{0 k}\right|^{2}$ with the characteristic width $\Delta k \simeq 1$, which implies that the typical spatial size of the initial random fluctuations is also of the order of unity and much larger than the internal coherence length (that is, $\varepsilon$ ). Such random waves are often called partially coherent, particularly in the statistical optics context [36].

Given the 1D-NLSE evolution of individual realizations of the random field $\psi(x, t)$ the challenge is to determine the associated evolution of its statistical characteristics such as the PDF of the amplitude $|\psi|$, the power spectrum $\left|\psi_{k}\right|^{2}$, etc. The particular objective of this paper is to determine the short-time evolution of the normalized fourth moment $\kappa_{4}(t)$ defined as

$$
\kappa_{4}(t)=\frac{\left\langle|\psi(x, t)|^{4}\right\rangle}{\left\langle|\psi(x, t)|^{2}\right\rangle^{2}},
$$

where the brackets $\langle\ldots\rangle$ denote ensemble average performed over a large number of realizations of the random process $\psi(x, t)$. In what follows we shall be using the double average, $\left\langle\frac{1}{L} \int_{0}^{L}|\psi(x, t)|^{n} d x\right\rangle, n=2,4$, in Eq. (9) to compute $\kappa_{4}(t)$. This is implemented for the sake of convenience in the numerical simulations since, if the averaging procedure over space is not implemented, a very large statistical ensemble must be built, which leads to a large, unrealistic computational cost necessary to reach convergence of $\kappa_{4}(t)$.

The fourth moment (9) is an important characteristic of the PDF of a random process that quantifies the "heaviness" of its tail. In particular, it can be used to characterize the deviation from Gaussianity in the course of evolution, when the initial statistics is Gaussian, in which case $\kappa_{4}$ is known to be equal $2[35,37]$. The determination of $\kappa_{4}$, while providing limited information about the PDF as a whole, is particularly relevant to the rogue wave studies as the formation of a "heavy tail" of the PDF is associated with the frequent appearance of large-amplitude events in the random process' realizations $[7,9,20,35]$.

Figure 1 shows a typical initial evolution of a random wave in the regime where the cubic (Kerr) nonlinearity dominates linear dispersive effects, which corresponds to the semiclassical regime described by Eq. (1) with $\varepsilon \ll 1$ (in the numerical simulations we took $\varepsilon=0.1$ ). As shown in Fig. 1, the selffocusing dynamics tends to produce bright peaks while the self-defocusing dynamics leads to a decrease of the peak amplitudes but is accompanied by steepening of slopes in the random amplitude profile. While only the short-time evolution

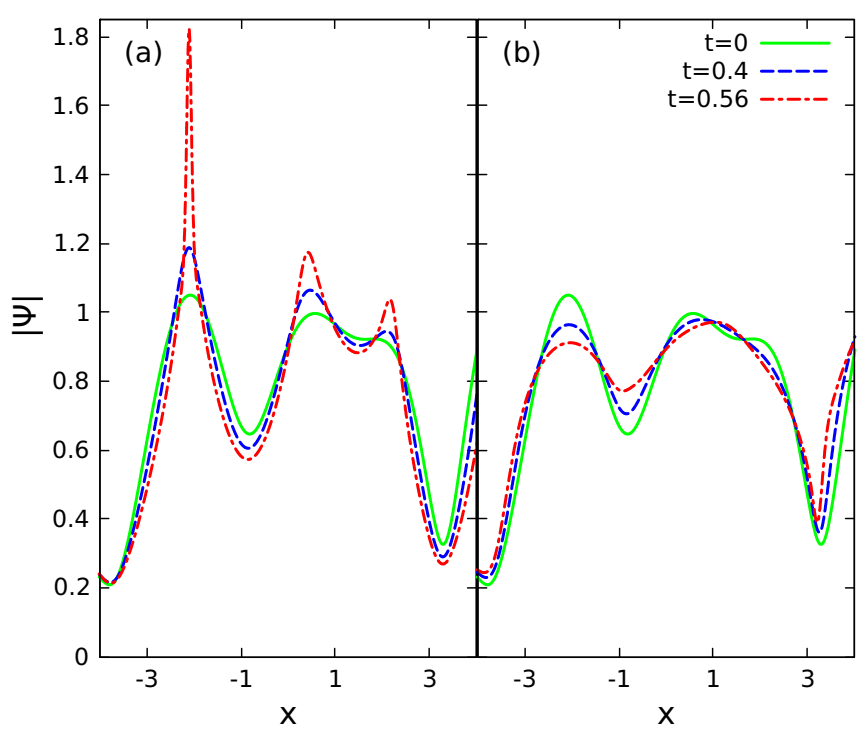

FIG. 1. Numerical simulations of Eq. (1) $(\varepsilon=0.1)$ showing the time evolution of a random field having Gaussian statistics at initial time $t=0$ (green solid lines). (a) Focusing regime $(\sigma=+1)$. At short evolution time $(t<0.56$ in the plot) the self-focusing dynamics produces bright peaks having the amplitude that grows in time. (b) Defocusing regime $(\sigma=-1)$. The self-defocusing dynamics induces the decrease in time of the amplitudes of random peaks. After some time (not reached in the plot), the random wave develops gradient catastrophes that are regularized by dispersive effects leading to the generation of breather structures in the focusing regime and of dispersive shock waves in the defocusing regime.

of the wave system is shown in Fig. 1, a longer development leads to the formation of gradient catastrophes-the explosion of the first derivatives of the wave's profile. These gradient catastrophes have qualitatively different geometrical nature in the defocusing regime (the wave-breaking singularity [38]) and the focusing regime (the elliptic umbilic singularity [39]). In both cases the gradient catastrophes are regularized by dispersive effects via the generation of nonlinear short wavelength oscillations: breather structures in the focusing regime [40] and dispersive shock waves in the defocusing regime (see Ref. [33] and references therein). For convenience, we shall call the initial nonlinear evolution preceding the formation of gradient catastrophes, the "prebreaking stage" in both defocusing and focusing regimes. The advantage of the semiclassical, dispersive-hydrodynamic approach employed in this paper is that it enables one to asymptotically separate the prebreaking and postbreaking stages of the evolution, which exhibit qualitatively different behaviors and require very different analytical methods for their descriptions.

The starting point of our analysis is the evolution of the nonlinear part $H_{\mathrm{NL}}$ of the Hamiltonian. Differentiating (8) we obtain

$$
\frac{d H_{\mathrm{NL}}}{d t}=\frac{\sigma}{L} \int_{0}^{L}|\psi|^{2}\left[\psi \frac{\partial \psi^{*}}{\partial t}+\psi^{*} \frac{\partial \psi}{\partial t}\right] d x .
$$

Using Eq. (1) and integrating by parts, one readily finds

$$
\frac{d H_{\mathrm{NL}}}{d t}=\frac{\sigma \varepsilon}{L} \int_{0}^{L} \operatorname{Im}\left[\left(\psi_{x} \psi^{*}\right)^{2}\right] d x .
$$


Now, using the Madelung transformation

$$
\psi=\sqrt{\rho} e^{i \frac{\phi}{\varepsilon}}, \quad u=\frac{\partial \phi}{\partial x},
$$

Eq. (11) can be rewritten as

$$
\frac{d H_{\mathrm{NL}}}{d t}=\frac{\sigma}{L} \int_{0}^{L} \rho u \rho_{x} d x .
$$

Noticing from (9), (4), and (8) that

$$
\kappa_{4}(t)=\frac{2\left\langle H_{\mathrm{NL}}\right\rangle}{\sigma\langle N\rangle^{2}}
$$

one obtains

$$
\frac{d \kappa_{4}}{d t}=\frac{2}{\sigma\langle N\rangle^{2}} \frac{d\left\langle H_{\mathrm{NL}}\right\rangle}{d t}=\frac{2}{\langle N\rangle^{2} L} \int_{0}^{L}\left\langle\rho u \rho_{x}\right\rangle d x .
$$

We now derive an analytical expression for $\kappa_{4}(t)$ for short evolution times, $t \ll 1$. If the dispersion parameter is small, $\varepsilon \ll 1$, the initial dynamics are dominated by nonlinearity. To describe these dynamics analytically, we consider the semiclassical limit of the 1D-NLSE (1) which is found by applying the Madelung transform (12) and letting $\varepsilon \rightarrow 0$. Assuming smooth evolution of $\rho(x, t)$ and $u(x, t)$ in the prebreaking regime, we obtain in the limit $\varepsilon \rightarrow 0$ the following well-known set of nonlinear geometric optics equations [32, 41-44],

$$
\begin{aligned}
& \rho_{t}+(\rho u)_{x}=0 \\
& u_{t}+u u_{x}-\sigma \rho_{x}=0 .
\end{aligned}
$$

If $\sigma=-1$, then Eqs. (16) are identical to the shallow-water equations for an incompressible fluid with $\rho>0$ and $u$ interpreted as the fluid depth and the depth-averaged horizontal fluid velocity, respectively. In the nonlinear fiber optics context, $\rho$ represents the instantaneous optical power and $u$ represents the instantaneous frequency (or chirp) [45].

Rigorous proofs of the pointwise convergence, as $\varepsilon \rightarrow 0$, of solutions of the 1D-NLSE (1) to the solutions of the dispersionless system (16) with the same initial data, prior to the formation of gradient catastrophe, can be found for certain classes of initial data in Ref. [46] (defocusing) and in Refs. [47,48] (focusing). Some important particular exact solutions of system (16) for the focusing case have been found as early as the 1960s and 1970s (see Refs. [49-51]). A detailed mathematical analysis of the prebreaking dynamics in the defocusing case can be found in Ref. [41] (see also Ref. [52] for the special case of the wave breaking into vacuum).

It follows from the above consideration that, to study the prebreaking dynamics of partially coherent waves in 1DNLSE (1), we need to be able to describe random solutions of system (16) obtained by evolving initial data $\rho(x, 0)$, $u(x, 0)$ with given statistics (e.g., corresponding to the Gaussian statistics of $\psi)$. The study of such solutions has been recently initiated for the defocusing case in the context of the interaction of random Riemann waves in fiber optics [32] (see also Ref. [13]). In this connection one must stress that the term "prebreaking dynamics" is understood here in the probabilistic sense, as for random initial data there is always a nonzero probability of having gradient catastrophe at any, arbitrarily small, moment of time. However, due to initial data having typical size $\Delta x=\mathcal{O}(1)$, we assume that for small $\varepsilon$

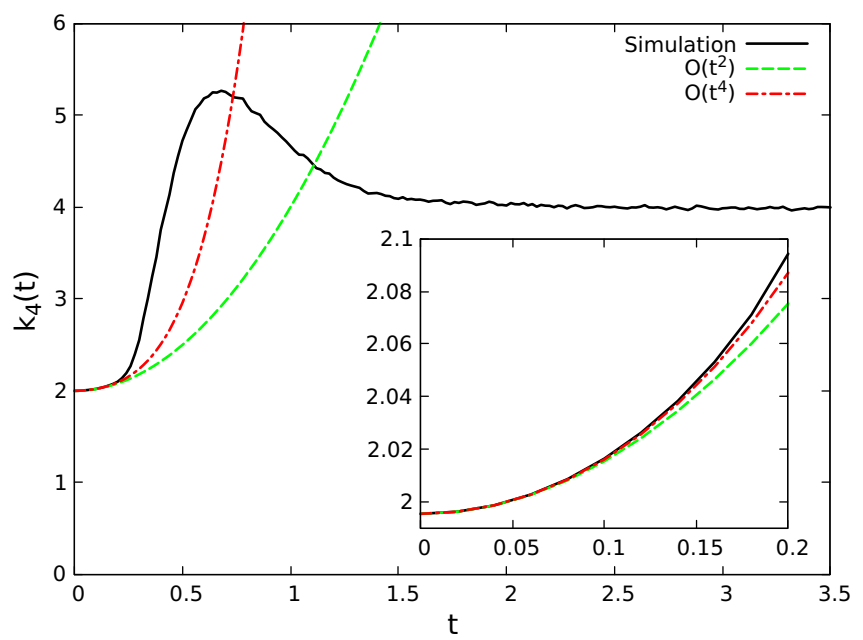

FIG. 2. Black solid line: Numerical simulations of Eq. (1) with $\varepsilon=0.1$ in the focusing regime $(\sigma=+1)$ showing the time evolution of the normalized fourth-order moment of the random field having at $t=0$ the Gaussian statistics and the Fourier spectrum defined by Eq. (26) with $\Delta k=1, N=1$. Green dashed line: Analytical result given by Eq. (27) at leading order in $t^{2}$. Red dashed-dotted line: Analytical result given by Eq. (27) including $t^{2}$ and $t^{4}$ evolution terms. The inset shows an enlarged view of the evolution of $\kappa_{4}(t)$ for $0<t<0.2$.

the contribution of such early gradient catastrophes to the statistics is negligibly small.

To this end, with the short-time, prebreaking evolution in mind, we look for the solutions of Eqs. (16) in the form of the time power-series expansions for the realizations $\rho(x, t)$ and $u(x, t)$ :

$$
\begin{aligned}
& \rho(x, t)=\rho_{0}(x)+\rho_{1}(x) t+\rho_{2}(x) t^{2}+\rho_{3}(x) t^{3}+\mathcal{O}\left(t^{4}\right) \\
& u(x, t)=u_{1}(x) t+u_{2}(x) t^{2}+u_{3}(x) t^{3}+\mathcal{O}\left(t^{4}\right)
\end{aligned}
$$

These time power-series expansions provide the description of the evolution of $\rho$ and of $u$ over timescales shorter than the typical time $t^{*}$ at which the gradient catastrophes occur. Rigorously speaking $t^{*}$ depends on the exact shapes of the initial bell-shaped pulses found in the initial random field but for partially coherent waves with the typical amplitude and width of individual pulses equal to $1, t^{*}$ is typically around 0.5 , as shown in Ref. [40]. Our theoretical analysis is therefore restricted to evolution times shorter than $t^{*} \approx 0.5$. This is well illustrated by Figs. 2 and 3 that show that there is a very good quantitative agreement between numerical simulations of Eq. (1) and our analytical results between $t=0$ and $t=0.2$.

Moreover, it is important to note that the term $\mathcal{O}\left(t^{4}\right)$ is the error between the Taylor expansion and the solution of the model (16). We derive here a solution of the zero-dispersion limit of $1 D-N L S E(\epsilon \rightarrow 0)$ for short time. The neglected term in (16) is $\mathcal{O}\left(\epsilon^{2}\right)$. Thus, for example, for small but finite values of $\epsilon$, the second order of the Taylor expansion in time of $\rho(t, x) \operatorname{reads}\left[\rho_{2}(x)+\mathcal{O}\left(\epsilon^{2}\right)\right] t^{2}$. We show in Sec. III that the results obtained in the zero-dispersion limit are robust and are in good agreement with simulations of 1D-NLSE for $\epsilon=0.1$. 


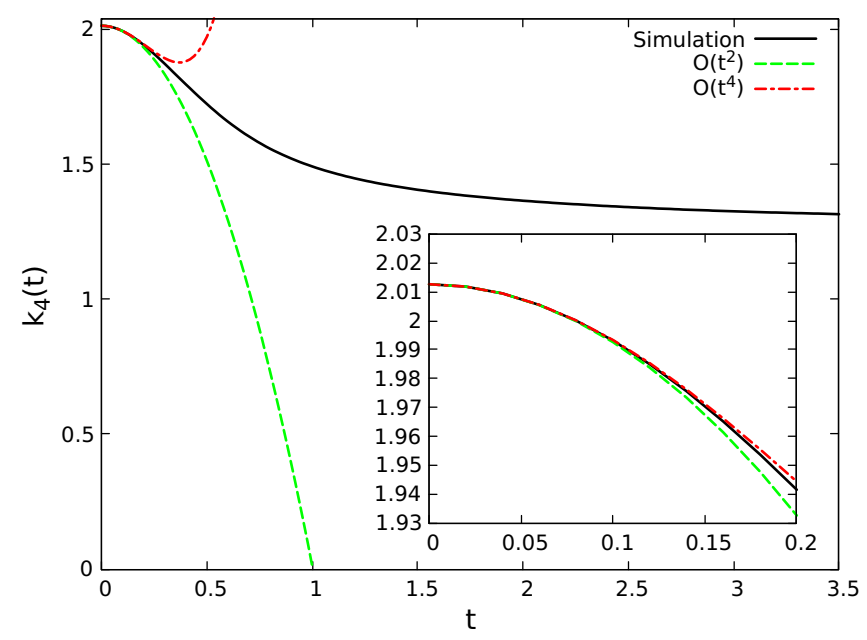

FIG. 3. Black solid line: Numerical simulations of Eq. (1) with $\varepsilon=0.1$ in the defocusing regime $(\sigma=-1)$ showing the time evolution of the normalized fourth-order moment of the random field having at $t=0$ the Gaussian statistics and the Fourier spectrum defined by Eq. (26) with $\Delta k=1, N=1$. Green dashed line: Analytical result given by Eq. (27) at leading order in $t^{2}$. Red dasheddotted line: Analytical result given by Eq. (27) including $t^{2}$ and $t^{4}$ evolution terms. The inset shows an enlarged view of $\kappa_{4}(t)$ plot for $0<t<0.2$.

In (17) we assumed that initially, $u(x, 0)=u_{0}=0$, which agrees with typical physical condition $u_{0} \ll \rho_{0}$ satisfied in standard realistic experimental conditions. Indeed timescales of amplitude and phase in partially coherent waves are generally similar $[\mathcal{O}(1)$ here]. Considering the normalizations given by the Eq. (12), this means that the derivative of the phase $\frac{\partial\left(\phi_{0} / \varepsilon\right)}{\partial x}=\mathcal{O}(1)$ and thus $u_{0}=\frac{\partial \phi_{0}}{\partial x}=\mathcal{O}(\varepsilon)$, whereas $\rho_{0}=\mathcal{O}(1)$. This assumption is for example satisfied in the experiments on the propagation of partially coherent light through optical fibers, see Ref. [32].

Substituting Eqs. (17) into Eqs. (16) we obtain

$$
\begin{aligned}
& \rho(x, t)=\rho_{0}-\frac{1}{4} \sigma\left[\rho_{0}^{2}\right]_{x x} t^{2}+\mathcal{O}\left(t^{4}\right), \\
& u(x, t)=\sigma \rho_{0 x} t-\left(\frac{1}{12}\left[\rho_{0}^{2}\right]_{x x x}+\frac{1}{3} \rho_{0 x} \rho_{0 x x}\right) t^{3}+\mathcal{O}\left(t^{4}\right) .
\end{aligned}
$$

Next, substituting Eqs. (18) into Eq. (15) and integrating in time, we obtain the following expression for the time evolution of the normalized fourth moment of the field amplitude:

$$
\begin{aligned}
\kappa_{4}(t)-\kappa_{4}(0)= & \frac{\sigma t^{2}}{\langle N\rangle^{2} L} \int_{0}^{L}\left\langle\rho_{0} \rho_{0 x}^{2}\right\rangle d x \\
& -\frac{t^{4}}{2\langle N\rangle^{2} L} \int_{0}^{L}\left\langle\frac{2}{3} \rho_{0}^{2} \rho_{0 x} \rho_{0 x x x}\right. \\
& \left.+\frac{17}{6} \rho_{0} \rho_{0 x}^{2} \rho_{0 x x}+\frac{1}{2} \rho_{0 x}^{4}\right\rangle d x+\mathcal{O}\left(t^{6}\right) .
\end{aligned}
$$

Equation (19) is our main general result. We note that it can also be obtained by a direct substitution of the expansion of $\rho=|\psi|^{2}$ in the kurtosis formula (9). We note, however, that this would require computing the terms $\mathcal{O}\left(t^{4}\right)$ in the expansion
(18) for $\rho$, which is avoided here by using in Eq. (15) the $\mathcal{O}\left(t^{3}\right)$ terms in the related expansion for $u$ ensuring the necessary $\mathcal{O}\left(t^{4}\right)$ accuracy in Eq. (19).

One can make now two important observations. The first one is that Eq. (19) shows that the normalized fourth-order moment $\kappa_{4}(t)$ of the field evolves quadratically with time at leading order for $t \ll 1$. The second observation is that Eq. (19) shows that the increasing or decreasing nature of the time evolution of $\kappa_{4}(t)$ is determined by the value taken by $\sigma$. In the focusing regime $(\sigma=+1), \kappa_{4}(t)$ is an increasing function of time which means that the nonlinear evolution of the wave field is characterized by PDFs that exhibit heavy tailed deviations from the initial statistical distribution. On the other hand, in the defocusing regime $(\sigma=-1) \kappa_{4}(t)$ becomes a decreasing function of time which implies low-tailed deviations from the initial statistics occurring in this regime. The statistical features described by Eq. (19) are in full qualitative agreement with the results that have been recently obtained in numerical and experimental investigations of integrable turbulence [7-9,12,13,26].

Let us emphasize that the decreasing or increasing nature of the time evolution of $\kappa_{4}$ has also been shown to be determined by the defocusing or focusing nature of the propagation regime for weakly nonlinear dispersive random waves that are described by the 1D-NLSE [28]. Theoretical approaches that have been used in the weakly nonlinear regime are based on the wave turbulence theory and they consist in deriving quasikinetic equations for the lowest-order moments of the wave field $[28,30]$. Dispersion plays crucial role in that consideration. Our work is based on a completely different, dispersive-hydrodynamic approach, where dispersive effects are initially not of dominant but of perturbative nature.

\section{INITIAL CONDITIONS WITH GAUSSIAN STATISTICS}

Equation (19) represents a general result that is derived with the only assumption that $\varepsilon \ll 1$. As we already stressed, it is valid before the typical time of the gradient catastrophe occurrence, i.e., for $t \ll 1$ [for random initial conditions with typical scales for $\rho$ and $x$ variations at $\mathcal{O}(1)]$. Importantly, Eq. (19) is derived without any assumption on the nature of the initial statistics of the random wave field. In this section we show that Eq. (19) can be further simplified if the random wave field taken as initial condition has Gaussian statistics. To this end, we assume that the random initial field $\psi(x, 0)$ is composed of a linear superposition of a large number of independent random Fourier modes $\psi_{k}(t=0)=\psi_{0 k}=\left|\psi_{0 k}\right| e^{i \phi_{0 k}}$, so that by the central limit theorem $\psi(x, 0)$ is a Gaussian random field [3].

In the random-phase and amplitude model, $\left|\psi_{0 k}\right|$ and $\phi_{0 k}$ are both taken as randomly distributed variables [3]. Here we will mainly use the so-called random-phase (RP) model in which only the phases $\phi_{0 k}$ of the Fourier modes are considered as being random [3]. In this model, the phase of each Fourier mode is randomly and uniformly distributed between $-\pi$ and $\pi$. Moreover, the phases of separate Fourier modes are assumed to be uncorrelated so that $\left\langle e^{i \phi_{0 k}} e^{i \phi_{0 k^{\prime}}}\right\rangle=\delta_{k}^{k^{\prime}}$. In the above expression, the brackets, as usual, represent the averaging over an ensemble of many realizations of the random process; $\delta_{k}^{k^{\prime}}$ is the Kronecker symbol defined by $\delta_{k}^{k^{\prime}}=1$ if 
$k=k^{\prime}$ and $\delta_{k}^{k^{\prime}}=0$ if $k \neq k^{\prime}$. With the assumptions of the RP model described above, the statistics of the initial field is homogeneous, which means that all statistical moments of the initial complex field $\psi(x, t=0)=\psi_{0}(x)$ do not depend on $x[15,53]$. This RP description of the initial random field has been shown to describe in a satisfatory way many experiments performed in the field of integrable turbulence [7-9,12,13,18,19,26,54].

Given the $\delta$ correlation of the random phases, the second moment of a field composed from the linear superposition of a large number of independent Fourier components having Gaussian statistics is readily evaluated as

$$
\left\langle\psi_{k} \psi_{k^{\prime}}^{*}\right\rangle=n_{k} \delta_{k}^{k^{\prime}}
$$

and the sixth moment can be factored into products of the second moments by using Wick's decomposition [3]

$$
\begin{aligned}
& \left\langle\psi_{k_{1}} \psi_{k_{2}} \psi_{k_{3}} \psi_{k_{4}}^{*} \psi_{k_{5}}^{*} \psi_{k_{6}}^{*}\right\rangle \\
& =n_{k_{1}} n_{k_{2}} n_{k_{3}}\left[\delta_{k_{4}}^{k_{1}} \delta_{k_{5}}^{k_{2}} \delta_{k_{6}}^{k_{3}}+\delta_{k_{4}}^{k_{1}} \delta_{k_{5}}^{k_{3}} \delta_{k_{6}}^{k_{2}}+\delta_{k_{4}}^{k_{2}} \delta_{k_{5}}^{k_{1}} \delta_{k_{6}}^{k_{3}}\right. \\
& \left.\quad+\delta_{k_{4}}^{k_{2}} \delta_{k_{5}}^{k_{3}} \delta_{k_{6}}^{k_{1}}+\delta_{k_{4}}^{k_{3}} \delta_{k_{5}}^{k_{1}} \delta_{k_{6}}^{k_{2}}+\delta_{k_{4}}^{k_{3}} \delta_{k_{5}}^{k_{2}} \delta_{k_{6}}^{k_{1}}\right] .
\end{aligned}
$$

Now, using Eq. (21), one can evaluate the coefficient for the $\mathcal{O}\left(t^{2}\right)$ term in the expansion (19):

$$
\begin{aligned}
& \frac{\sigma}{\langle N\rangle^{2} L} \int_{0}^{L}\left\langle\rho_{0} \rho_{0 x}^{2}\right\rangle d x \\
& =\frac{\sigma}{\langle N\rangle^{2}} \sum_{k_{1}, \ldots k_{6}}\left(\frac{2 i \pi}{L}\right)^{2} \delta_{k_{4}+k_{5}+k_{6}}^{k_{1}+k_{2}+k_{3}} \\
& \quad \times\left\langle\psi_{0 k_{1}} \psi_{0 k_{2}} \psi_{0 k_{3}} \psi_{0 k_{4}}^{*} \psi_{0 k_{5}}^{*} \psi_{0 k_{6}}^{*}\right\rangle\left(k_{2}-k_{5}\right)\left(k_{3}-k_{6}\right),
\end{aligned}
$$

where we have used the notation $\psi_{0 k_{i}}=\psi_{k_{i}}(0)$ for the Fourier component at $t=0$. Using Eq. (21), we obtain the following expression for the short time evolution of the fourth-order moment of a random wave field that has Gaussian statistics at initial time, i.e., $\kappa_{4}(0)=2$ :

$$
\begin{aligned}
\kappa_{4}(t)-\kappa_{4}(0)= & -\frac{\sigma}{\langle N\rangle^{2}} \sum_{k_{1}, k_{2}, k_{3}} n_{0 k_{1}} n_{0 k_{2}} n_{0 k_{3}}\left(\frac{2 \pi}{L}\right)^{2} \\
& \times\left[-2\left(k_{2}-k_{3}\right)^{2}\right] t^{2}+\mathcal{O}\left(t^{4}\right),
\end{aligned}
$$

where $n_{0 k_{i}}=n_{k_{i}}(0)$ are the components of the power spectrum at $t=0$. Using Eqs. (5), (4), and (7) and taking into account that $P=0$ for our random Gaussian field, we can finally rewrite Eq. (23) as

$$
\kappa_{4}(t)-\kappa_{4}(0)=8 \sigma\left\langle H_{L}(0)\right\rangle t^{2}+\mathcal{O}\left(t^{4}\right) .
$$

[Note that $H_{L}=\mathcal{O}(1)$ and $H_{\mathrm{NL}}=\mathcal{O}(1)$, whereas the linear part of the Hamiltonian (6) is $\left.\mathcal{O}\left(\varepsilon^{2}\right)\right]$.

A similar, but somewhat lengthy, calculation permits one to obtain a more accurate expression that includes $\mathcal{O}\left(t^{4}\right)$ correction (see Appendix):

$$
\begin{aligned}
\kappa_{4}(t)-\kappa_{4}(0)= & 8 \sigma\left\langle H_{L}(0)\right\rangle t^{2}+\left[\frac{208}{3}\left\langle H_{L}(0)\right\rangle^{2}\right. \\
& \left.+4\langle N\rangle\left\langle\sum_{k}\left(\frac{2 \pi k}{L}\right)^{4}\left|\psi_{0 k}\right|^{2}\right\rangle\right] t^{4}+\mathcal{O}\left(t^{6}\right) .
\end{aligned}
$$

Equations (24) and (25) show that the time evolution of the fourth moment of the initially Gaussian random wave field is determined by the linear part $H_{L}(0)$ of the Hamiltonian computed for the initial condition.

Equation (25) can be further simplified if we assume that the shape of the Fourier power spectrum of the initial random field is described by a Gaussian,

$$
\left|\psi_{0 k}\right|^{2}=n_{0} e^{-\frac{k^{2}}{(\Delta k)^{2}}}
$$

The amplitude $n_{0} \in \mathbb{R}^{+}$has to be determined from the normalization condition provided by Eq. (4). The linear energy density determined from Eq. (7) is $\left\langle H_{L}\right\rangle=\frac{N(\Delta k)^{2}}{4}$, and we can finally rewrite Eq. (25) only in terms of the density of particles (or optical power) $N$ and of the width $\Delta k$ of the initial Fourier spectrum,

$$
\kappa_{4}(t)-\kappa_{4}(0)=2 \sigma N(\Delta k)^{2} t^{2}+\frac{22 N^{2}(\Delta k)^{4}}{3} t^{4}+\mathcal{O}\left(t^{6}\right) .
$$

\section{NUMERICAL SIMULATIONS}

In this section, we use numerical simulations of Eq. (1) to investigate the range and the degree of validity of the semiclassical approach to the statistics of integrable turbulence presented in Secs. II and III. The initial condition used in our numerical simulations is a random complex field having Gaussian statistics. The amplitudes of the Fourier components are taken to be distributed according to Eq. (26). In our numerical simulations, the spectral phases $\phi_{0 k}$ are random, statistically independent real numbers, uniformly distributed between $-\pi$ and $+\pi$. The width $\Delta k$ of the initial spectrum profile (26) is taken to be unity $(\Delta k=1)$, and the value of $\varepsilon$ in (1) is taken to be 0.1 . The numerical simulations are performed by using a pseudospectral method with the numerical box having size $L=256$ for the defocusing regime and $L=128$ in the focusing regime that is discretized by using $2^{16}$ points.

Figure 2 shows the time evolution of the normalized fourth moment $\kappa_{4}(t)$ of the random wave field in the focusing regime $(\sigma=+1)$. The curve plotted with black line represents the result of the numerical simulation of Eq. (1). In the large box limit $(L \rightarrow \infty)$, the value assumed by $\kappa_{4}$ at $t=0$ should be exactly 2 . As can be seen from Fig. 2 , in the numerical experiments the value taken by $\kappa_{4}(t)$ at $t=0$ slightly differs from 2 [see the inset in Fig. (2)] because the conditions of the central limit theorem are not perfectly fulfilled in our numerical simulations. Indeed, because of the finite value of $L$, the number of Fourier modes in the spectrum given by Eq. (26) is finite, in particular, in the full width at half maximum (FWHM) we count $\frac{\sqrt{2 \ln 2} L}{\pi}$ modes. Importantly, the deviation of the initial condition from Gaussian statistics affects only the value of $\kappa_{4}(0)$ in Eq. (21) but not the evolution. In the initial (before the formation of a gradient catastrophe) stage of the nonlinear evolution of the random wave, $\kappa_{4}(t)$ is at first an increasing function of time that later reaches a maximum around $t \sim 0.6$. Then $\kappa_{4}(t)$ becomes a decaying function of time that reaches a stationary value around $\sim 4$ at long evolution time. A similar evolution of $\kappa_{4}(t)$ has already been evidenced in numerical simulations presented in Ref. [35]. 
The occurrence of the maximum of $\kappa_{4}(t)$ has been linked in Ref. [55] to the formation of the Peregrine breathers as the universal local structures regularizing gradient catastrophes in the semiclassical focusing 1D-NLSE [34,40].

The curves plotted with green (dashed) and red (dasheddotted) lines in Fig. 2 show monotonic evolutions of $\kappa_{4}(t)$ that are obtained from Eq. (27). In particular the curves plotted in the inset of Fig. 2 clearly reveal a very good quantitative agreement between numerical and theoretical results. In particular, a better agreement between numerics and theory is obtained by including the fourth-order correction term found in the time expansion of the solution, see Eq. (27). A significant quantitative disagreement is found between our theoretical results and the numerical simulation at evolution times greater than $\sim 0.2$. This arises from the fact that our approach is only valid at evolution times that are shorter that the typical wave breaking time (the prebreaking description). The significant occurrence of wave breakings at evolution times greater than $\sim 0.2$ has strong influence on the wave evolution and subsequently the wave statistics in a way that cannot be accounted for by using our prebreaking treatment.

Figure 3 shows the comparison between the numerical simulation of Eq. (1) and the theoretical result given by Eq. (27) in the defocusing regime $(\sigma=-1)$. In the defocusing regime, $\kappa_{4}(t)$ is a monotonically decreasing function of time, as already evidenced in Ref. [35]. As for the focusing regime, a very good quantitative agreement is obtained between numerics and the theory at short evolution time $(t<0.2)$, i.e., before the typical occurrence of gradient catastrophes.

While the developed theory is rigorously valid in the semiclassical limit as $\varepsilon \rightarrow 0$ Fig. 2 and Fig. 3 provide the evidence of a good quantitative agreement between numerical simulations and our theory for $\varepsilon=0.1$. To further verify the robustness of our results we have performed further numerical simulations for $\varepsilon$ ranging between 0.08 and 0.5 . In all cases a very good quantitative agreement was obtained between the numerics and the theory at short evolution time $(t<0.2)$.

\section{CONCLUSION}

In this paper, we have undertaken an analytical study of the problem of the evolution of a random wave field in the 1D-NLSE for both focusing and defocusing regimes. This has been done from the perspective of dispersive hydrodynamics, a semiclassical theory of nonlinear dispersive waves exhibiting two distinct spatiotemporal scales: the long scale specified by initial conditions and the short scale by the internal coherence length (i.e., the typical size $\varepsilon$ of the coherent structures) [31]. This scale separation enabled us to split the time evolution of the nonlinear random wave system (integrable turbulence) into the initial, "prebreaking" stage, preceding the formation of gradient catastrophes, when the evolution of the 1D-NLSE wave field is almost everywhere smooth, and the "postbreaking" stage characterized by the generation of short-scale nonlinear oscillations (breathers or dispersive shock waves depending on the focusing vs. defocusing character of the 1D-NLSE).
Our work is concerned with the initial, prebreaking stage of the semiclassical integrable turbulence, when the dynamical and statistical features can be analytically described in terms of random solutions of the dispersionless (nonlinear geometric optics) system (16). As a result, we have derived a simple asymptotic formula describing the evolution of the normalized fourth moment of the random wave field. This formula, applied to the problem of the 1D-NLSE evolution of random field having initial Gaussian statistics, describes the initial stage of the formation of heavy tails of the PDF of the field amplitude in the focusing case and the formation of low tails in the defocusing case.

Recently, an exact and general identity that relates the changes in the statistical properties of the wave field to the changes of its Fourier spectrum has been derived by using the Hamiltonian structure of 1D-NLSE [35]. In other words, the knowledge of the fourth-order moment also provides the description of spectral properties. The general description of the stationary state of integrable turbulence and the theoretical prediction of the fourth-order moment is still an open fundamental question. In the weakly nonlinear regime, the wave turbulence approach provides a statistical description of the nonlinear propagation of random wave fields in 1D-NLSE systems [28-30,56]. Recently, using an approach based on the so-called large deviation theory, it has been shown that rogue waves obey a large deviation principle, i.e., the heavy tails of the PDF of the random wave field are dominated by single realizations $[57,58]$. This approach is very promising but does not provide a simple formula for the evolution of the statistics. In this article we have demonstrated that the semiclassical approach is an extremely powerful tool enabling one to describe in a simple way the early stage of integrable turbulence in the strongly nonlinear (or small dispersion) regime. The proposed methodology can be applied to the description of partially coherent random nonlinear waves described by other integrable equations, including shallow water waves described by the KdV equation and its extensions. In particular, the prebreaking statistics of bidirectional random shallow water waves is equivalent to that described by the defocusing 1D-NLSE and studied in this paper.

The semiclassical approach to the statistics of random waves in integrable systems is general and can be used beyond the short-time asymptotic regime. It is known very well that, in the semiclassical limit the evolution of nonlinear dispersive waves after the gradient catastrophe point is described by the so-called Whitham modulation equations [59] governing the behavior of the averaged integrals of motion, and replacing the dispersionless system (16) (see Refs. [33,60-62] and references therein for the application of the Whitham theory to the defocusing and focusing 1D-NLSE). Such an extension of the proposed method to longer times is very promising but also highly nontrivial.

\section{ACKNOWLEDGMENTS}

This work was supported by EPSRC Grant No. EP/R00515X/2 (G.E.) and Dstl Grant No. DSTLX1000116851 (G.R., G.E., and S.R.). It has also been partially supported by the Agence Nationale de la Recherche through 
the LABEX CEMPI project (ANR-11-LABX-0007) and by the Ministry of Higher Education and Research, Hauts-DeFrance Regional Council and European Regional Development Fund (ERDF) through the Contrat de Projets Etat-
Région (CPER Photonics for Society P4S). G.E. and G.R. thank the PhLAM laboratory at the University of Lille for hospitality and partial financial support (G.E.). The authors thank T. Congy for some clarifying comments.

\section{APPENDIX: COMPUTATION OF $\mathcal{O}\left(t^{4}\right)$ CORRECTIONS FOR THE CASE OF GAUSSIAN STATISTICS AT $t=0$}

Here we provide the simplified expressions for the three terms that are found in the integral giving the coefficient of the $O\left(t^{4}\right)$ term in Eq. (19). To obtain these expressions, we assume Gaussian statistics at the initial time and use Eq. (20) and Eq. (21) to obtain

$$
\begin{aligned}
-\frac{1}{3\langle N\rangle^{2} L} \int_{0}^{L}\left\langle\rho_{0}^{2} \rho_{0 x} \rho_{0 x x x}\right\rangle d x= & -\frac{1}{3\langle N\rangle^{2}}\left(\frac{2 i \pi}{L}\right)^{4} \sum_{k_{1}, \ldots k_{6}}\left\langle\psi_{0 k_{1}} \psi_{0 k_{2}} \psi_{0 k_{3}} \psi_{0 k_{4}} \psi_{0 k_{5}}^{*} \psi_{0 k_{6}}^{*} \psi_{0 k_{7}}^{*} \psi_{0 k_{8}}^{*}\right\rangle \\
& \times \delta_{k_{5}+k_{6}+k_{7}+k_{8}}^{k_{1}+k_{2}+k_{3}+k_{4}}\left(k_{3}-k_{7}\right)\left(k_{4}-k_{8}\right)^{3}=48\left\langle H_{L}\right\rangle^{2}+4\langle N\rangle\left\langle\sum_{k}\left(\frac{2 \pi k}{L}\right)^{4}\left|\psi_{0 k}\right|^{2}\right\rangle \\
-\frac{17}{12\langle N\rangle^{2} L} \int_{0}^{L}\left\langle\rho_{0} \rho_{0 x}^{2} \rho_{0 x x}\right\rangle d x= & -\frac{17}{12\langle N\rangle^{2}}\left(\frac{2 i \pi}{L}\right)^{4} \sum_{k_{1}, \ldots k_{6}}\left\langle\psi_{0 k_{1}} \psi_{0 k_{2}} \psi_{0 k_{3}} \psi_{0 k_{4}} \psi_{0 k_{5}}^{*} \psi_{0 k_{6}}^{*} \psi_{0 k_{7}}^{*} \psi_{0 k_{8}}^{*} \delta_{k_{5}+k_{6}+k_{7}+k_{8}}^{k_{1}+k_{2}+k_{3}+k_{4}}\right. \\
& \times\left(k_{2}-k_{6}\right)\left(k_{3}-k_{7}\right)\left(k_{4}-k_{8}\right)^{2}=\frac{136}{3}\left\langle H_{L}\right\rangle^{2}, \\
-\frac{1}{4\langle N\rangle^{2} L} \int_{0}^{L}\left\langle\rho_{0 x}^{4}\right\rangle d x= & \frac{1}{4\langle N\rangle^{2}}\left(\frac{2 i \pi}{L}\right)^{4} \sum_{k_{1}, \ldots k_{6}}\left\langle\psi_{0 k_{1}} \psi_{0 k_{2}} \psi_{0 k_{3}} \psi_{0 k_{4}} \psi_{0 k_{5}}^{*} \psi_{0 k_{6}}^{*} \psi_{0 k_{7}}^{*} \psi_{0 k_{8}}^{*} \delta_{k_{5}+k_{6}+k_{7}+k_{8}}^{k_{1}+k_{2}+k_{3}+k_{4}}\right. \\
& \times\left(k_{1}-k_{5}\right)\left(k_{2}-k_{6}\right)\left(k_{3}-k_{7}\right)\left(k_{4}-k_{8}\right)=-24\left\langle H_{L}\right\rangle^{2} .
\end{aligned}
$$

[1] U. Frisch, Turbulence, the Legacy of A. N. Kolmogorov (Cambridge University Press, Cambridge, UK, 1995).

[2] V. Zakharov, V. L'vov, and G. Falkovich, Kolmogorov Spectra of Turbulence I (Springer, Berlin, 1992).

[3] S. Nazarenko, Wave Turbulence, Lecture Notes in Physics (Springer, Berlin, 2011).

[4] V. E. Zakharov, Stud. Appl. Math. 122, 219 (2009).

[5] V. E. Zakharov and A. A. Gelash, Phys. Rev. Lett. 111, 054101 (2013).

[6] D. Agafontsev and V. E. Zakharov, Nonlinearity 28, 2791 (2015).

[7] P. Walczak, S. Randoux, and P. Suret, Phys. Rev. Lett. 114, 143903 (2015).

[8] P. Suret, R. El Koussaifi, A. Tikan, C. Evain, S. Randoux, C. Szwaj, and S. Bielawski, Nat. Commun. 7, 13136 (2016).

[9] S. Randoux, P. Walczak, M. Onorato, and P. Suret, Physica D 333, 323 (2016).

[10] J. M. Soto-Crespo, N. Devine, and N. Akhmediev, Phys. Rev. Lett. 116, 103901 (2016).

[11] N. Akhmediev, J. M. Soto-Crespo, and N. Devine, Phys. Rev. E 94, 022212 (2016).

[12] S. Randoux and P. Suret, Integrable turbulence with nonlinear random optical waves, in Rogue and Shock Waves in Nonlinear Dispersive Media (Springer, Cham, 2016), pp. 277-307.

[13] P. Suret, E. Gennady, M. Onorato, and S. Randoux, Rogue waves in integrable turbulence: Semi-classical theory and fast measurements, in Nonlinear Guided Wave Optics (IOP, Bristol, UK, 2017), pp. 12-1-12-32.

[14] A. Tikan, S. Bielawski, C. Szwaj, S. Randoux, and P. Suret, Nat. Photon. 12, 228 (2018).
[15] A. Picozzi, J. Garnier, T. Hansson, P. Suret, S. Randoux, G. Millot, and D. Christodoulides, Phys. Rep. 542, 1 (2014).

[16] M. J. Ablowitz, M. Ablowitz, P. Clarkson, and P. A. Clarkson, Solitons, Nonlinear Evolution Equations and Inverse Scattering, Vol. 149 (Cambridge University Press, Cambridge, UK, 1991).

[17] C. Kharif, E. Pelinovsky, and A. Slunyaev, Rogue Waves in the Ocean (Springer Verlag, Berlin, 2009).

[18] M. Onorato, A. R. Osborne, M. Serio, L. Cavaleri, C. Brandini, and C. T. Stansberg, Phys. Rev. E 70, 067302 (2004).

[19] M. Onorato, A. Osborne, M. Serio, and L. Cavaleri, Phys. Fluids 17, 078101 (2005).

[20] M. Onorato, S. Residori, U. Bortolozzo, A. Montina, and F. Arecchi, Phys. Rep. 528, 47 (2013).

[21] D. R. Solli, C. Ropers, P. Koonath, and B. Jalali, Nature 450, 1054 (2007).

[22] A. Mussot, A. Kudlinski, M. Kolobov, E. Louvergneaux, M. Douay, and M. Taki, Opt. Express 17, 17010 (2009).

[23] D. Pierangeli, F. Di Mei, C. Conti, A. J. Agranat, and E. DelRe, Phys. Rev. Lett. 115, 093901 (2015).

[24] M. Närhi, B. Wetzel, C. Billet, S. Toenger, T. Sylvestre, J.-M. Merolla, R. Morandotti, F. Dias, G. Genty, and J. M. Dudley, Nat. Commun. 7, 13675 (2016).

[25] A. Safari, R. Fickler, M. J. Padgett, and R. W. Boyd, Phys. Rev. Lett. 119, 203901 (2017).

[26] S. Randoux, P. Walczak, M. Onorato, and P. Suret, Phys. Rev. Lett. 113, 113902 (2014).

[27] Y. Bromberg, U. Lahini, E. Small, and Y. Silberberg, Nat. Photon. 4, 721 (2010).

[28] P. A. E. M. Janssen, J. Phys. Oceanogr. 33, 863 (2003).

[29] D. B. S. Soh, J. P. Koplow, S. W. Moore, K. L. Schroder, and W. L. Hsu, Opt. Express 18, 22393 (2010). 
[30] P. Suret, A. Picozzi, and S. Randoux, Opt. Express 19, 17852 (2011).

[31] G. Biondini, G. El, M. Hoefer, and P. Miller, Physica D 333, 1 (2016).

[32] S. Randoux, F. Gustave, P. Suret, and G. El, Phys. Rev. Lett. 118, 233901 (2017).

[33] G. A. El and M. A. Hoefer, Physica D 333, 11 (2016).

[34] A. Tikan, C. Billet, G. El, A. Tovbis, M. Bertola, T. Sylvestre, F. Gustave, S. Randoux, G. Genty, P. Suret, and J. M. Dudley, Phys. Rev. Lett. 119, 033901 (2017).

[35] M. Onorato, D. Proment, G. El, S. Randoux, and P. Suret, Phys. Lett. A 380, 3173 (2016).

[36] L. Mandel and E. Wolf, Optical Coherence and Quantum Optics (Cambridge University Press, Cambridge, UK, 1995).

[37] R. El Koussaifi, A. Tikan, A. Toffoli, S. Randoux, P. Suret, and M. Onorato, Phys. Rev. E 97, 012208 (2018).

[38] Y. Pomeau, M. Le Berre, P. Guyenne, and S. Grilli, Nonlinearity 21, T61 (2008).

[39] B. Dubrovin, T. Grava, and C. Klein, J. Non. Sci. 19, 57 (2008).

[40] M. Bertola and A. Tovbis, Commun. Pure Appl. Math. 66, 678 (2013).

[41] M. G. Forest, C.-J. Rosenberg, and O. C. Wright, Nonlinearity 22, 2287 (2009).

[42] S. Wabnitz, C. Finot, J. Fatome, and G. Millot, Phys. Lett. A 377, 932 (2013).

[43] J. Fatome, C. Finot, G. Millot, A. Armaroli, and S. Trillo, Phys. Rev. X 4, 021022 (2014).

[44] Y. Kodama and S. Wabnitz, Opt. Lett. 20, 2291 (1995).

[45] B. Wetzel, D. Bongiovanni, M. Kues, Y. Hu, Z. Chen, S. Trillo, J. M. Dudley, S. Wabnitz, and R. Morandotti, Phys. Rev. Lett. 117, 073902 (2016).
[46] S. Jin, C. D. Levermore, and D. W. McLaughlin, Commun. Pure Appl. Math. 52, 613 (1999).

[47] S. Kamvissis, K. D.-R. McLaughlin, and P. D. Miller, Semiclassical Soliton Ensembles for the Focusing Nonlinear Schrodinger Equation (AM-154), 154 (Princeton University Press, Princeton, NJ, 2003).

[48] A. Tovbis, S. Venakides, and X. Zhou, Commun. Pure Appl. Math. 57, 877 (2004).

[49] V. I. Talanov, JETP Lett. 2, 138 (1965).

[50] S. A. Akhmanov, A. P. Sukhorukov, and R. V. Khokhlov, Sov. Phys. JETP 23, 1025 (1966).

[51] A. Gurevich and A. Shvartsburg, Sov. Phys. JETP 31, 1084 (1970).

[52] A. Moro and S. Trillo, Phys. Rev. E 89, 023202 (2014).

[53] A. Picozzi, Opt. Express 15, 9063 (2007).

[54] M. Onorato, A. R. Osborne, M. Serio, and S. Bertone, Phys. Rev. Lett. 86, 5831 (2001).

[55] A. Tikan, arXiv:1905.11938.

[56] N. Mori and P. A. E. M. Janssen, J. Phys. Oceanogr. 36, 1471 (2006).

[57] G. Dematteis, T. Grafke, and E. Vanden-Eijnden, Proc. Natl. Acad. Sci. U.S.A. 115, 855 (2018).

[58] G. Dematteis, T. Grafke, and E. Vanden-Eijnden, arXiv:1808.10764.

[59] G. Whitham, Linear and Nonlinear Waves (Wiley Interscience, New York, 1974), p. 636.

[60] A. M. Kamchatnov, Nonlinear Periodic Waves and Their Modulations: An Introductory Course (World Scientific, Singapore, 2000).

[61] G. A. El, E. G. Khamis, and A. Tovbis, Nonlinearity 29, 2798 (2016).

[62] A. Tovbis and G. A. El, Physica D 333, 171 (2016). 